\title{
Lemaitre's law and the spatial distributions of the cone photoreceptors in the retinas of vertebrates
}

\author{
Alireza Beygi \\ Centre for Information Services and High Performance Computing, \\ Technische Universität Dresden, Nöthnitzer Straße 46, 01062 \\ Dresden, Germany \\ Correspondence: alireza.beygi@tu-dresden.de
}

January 20, 2022

\begin{abstract}
By applying the principle of maximum entropy, we demonstrate the universality of the spatial distributions of the cone photoreceptors in the retinas of vertebrates. We obtain Lemaitre's law as a special case of our formalism.
\end{abstract}

Keywords: the principle of maximum entropy; cone photoreceptor mosaics

\section{Introduction}

The principle of maximum entropy provides an estimation for the underlying probability distribution of the observed data that corresponds best to the current available information about the system $[1,2]$. It has been used in fields as diverse as physics [3, 4], biology [5], ecology [4, 6], and natural language [7]. The philosophy behind this approach is to describe and predict the experimental observations by making the fewest number of assumptions (constraints), while it assumes no explicit underlying dynamics.

One of the main challenges in applying the principle of maximum entropy is to identify the constraints that should be imposed on the system. The authors of [8] recognized that, in the case which the experiments are repeatable, the expected value of the likelihood entropy is a relevant information that should be considered as a constraint; although, for a given system, its value is mostly unknown. In the present paper, we adopt this approach and recast it in the context of biology in order to be applicable to complex multicellular systems and develop it further. In a nutshell, we look for the configuration of biological receptors that maximizes the entropy, while the value of the extracellular microenvironmental 
entropy has been imposed as a constraint. This approach leads to a formalism which implies that configurations with lower microenvironmental entropy have a higher probability of occurrence and it allows us to interpret the involved free parameter (Lagrange multiplier) as a coarse-grained collective sensing, which quantifies how cells overall perceive their microenvironments. This idea that the organization of biological systems stems from an underlying optimization problem goes back to D'Arcy Thompson, which in his seminal work, On Growth and Form [9], he argues for the case of energy minimization, which leads, for example, to the prediction of cellular packing geometries in two-dimensional networks [10]. These geometric properties, obtained on the basis of the physical properties of the epithelial cells, can be considered as the factors that control the development and function of an organism [9]. In essence, here, we are replacing the energy minimization with the entropy maximization, with the advantage of ignoring the involved forces.

In this paper, we apply the aforementioned approach to the retinal cone photoreceptor mosaics. Cone photoreceptor cells are wavelength-sensitive receptors in the retinas of vertebrate eyes, which are responsible for color vision. The spatial distributions of these cells are commonly referred to as retinal cone photoreceptor mosaics [11]. Cone mosaic patterns vary among different species, which in each case it may reflect the evolutionary pressures that give rise to various adaptations to specific visual needs of a particular species with respect to its lifestyle; although, in most cases, the adaptive value of a particular cone mosaic is unknown [12]. From the perspective of the gene regulatory mechanisms, the most fundamental questions, such as: what are the mechanisms which control the mostly random distributions of the cone subtypes in the human retina? or, what migration mechanism determines the highly regular and ordered patterns of the cone subtypes in the retina of the zebrafish?, remain unanswered [13].

Our goal, here, is to establish a parameter, if it exists, which could play a role as a conserved quantity in the retinal patterning of different species. To this end, we employ the principle of maximum entropy, as explained earlier, without invoking any specific biological mechanisms. This allows us to identify a conserved coarse-grained retinal factor, called collective sensing, in divergent species of: rodent, dog, monkey, human, fish, and bird.

This paper is organized as follows: in Sec. 2, we formulate the entropy maximization problem; therein, we derive the virial equation of state for twodimensional cellular mosaics, known as Lemaitre's law, as a special case of our formalism. We apply the obtained formulas, in Sec. 3, to the spatial distributions of the cone photoreceptors in the retinas of different vertebrates and demonstrate the predictive power of our approach besides its explanatory nature. In Sec. 4, by using statistical analyses, we shed light on the obtained results. We summarize and conclude in Sec. 5. 


\section{Entropy maximization}

In statistical mechanics, in order to obtain the Boltzmann distribution from the principle of maximum entropy, one has to assume a constraint on the mean energy value; since in physics context, the expected value of the energy is a crucial information about the system. This approach leads to a formalism which temperature emerges as a free parameter and should be determined later from the experiment [14]. In a general setting, the challenge is to identify the relevant constraints. Caticha and Preuss [8] assumed a set of data generated by some experiment, where the only requirement was the experiment to be repeatable. If, for example, the experiment is performed twice, with the corresponding outcomes of $y_{1}$ and $y_{2}$, in the case that we discard the value of $y_{2}$, the resulting situation should be indistinguishable from if we had done the experiment only once. They argued that a constraint on the expected value of the entropy of the likelihood codifies this information. Inspired by this idea, we formulate our problem of the entropy maximization, suitable for multicelluar systems, as follows.

We denote the biological receptor as $x \in \mathcal{X}$ and the (extracellular) microenvironment, which could consist of chemicals or other cells, as $\mathcal{Y}$. We assume the following information about the system:

$$
\begin{gathered}
\sum_{x \in \mathcal{X}} p(x)=1, \\
S(Y \mid X)=\sum_{x \in \mathcal{X}} p(x) S(Y \mid X=x)=\bar{S},
\end{gathered}
$$

where the first one is a normalization condition, concerning the probability mass function of the receptors, and the second one implies the knowledge of the numerical value $\bar{S}$ of the microenvironmental entropy, $S(Y \mid X)$. Now, by the method of Lagrange multipliers, we maximize the Shannon entropy of the receptors, $S(X)=-\sum_{x \in \mathcal{X}} p(x) \ln p(x)$, while taking into account (1) and (2). The corresponding Lagrangian reads as

$$
L=S(X)-\lambda\left[\sum_{x \in \mathcal{X}} p(x)-1\right]-\beta\left[\sum_{x \in \mathcal{X}} p(x) S(Y \mid X=x)-\bar{S}\right]
$$

where $\lambda$ and $\beta$ are Lagrange multipliers. By solving $\partial L / \partial p(x)=0$, we obtain:

$$
p(x)=\frac{e^{-\beta S(Y \mid X=x)}}{Z}
$$

where $\lambda$ is absorbed in $Z$, also $S(Y \mid X=x)=-\sum_{y \in \mathcal{Y}} p(Y=y \mid X=$ $x) \ln p(Y=y \mid X=x)$, and $Z=\sum_{x \in \mathcal{X}} \exp [-\beta S(Y \mid X=x)]$. Equation (4) implies that receptors with lower microenvironmental entropy have a higher probability (assuming $\beta>0$ ); this will be confirmed in a particular biological setting in Sec. 3. 
A couple of remarks are in order. The application of the principle of maximum entropy strongly depends on how we specify the system configuration, which by itself depends on the nature of the problem at hand. In this paper (see Sec. 3), we are interested in the spatial distributions of the cone cells in the eye, where we assume there is a correlation (or, communication) between a cell and its nearest neighbor, and employ an appropriate spatial statistics to take this into account. Different ways of describing the system configuration of the same problem could lead to different results.

The second remark has to do with (2). Although, we have assumed the knowledge of $\bar{S}$, but we don't know its value in most cases, but rather, it is a quantity that its value should be known, thus we have formulated our problem as if we had this information [8]. By calculating the free parameter, $\beta$, from the experimental data, one can infer the value of $\bar{S}$. In general, since $\beta$ is introduced as the Lagrange multiplier for the entropy of the microenvironment given the set of receptors, i.e., $S(Y \mid X)$, we interpret $\beta$ as a coarse-grained collective sensing, that is, it assigns a number to how cells overall perceive their microenvironments, see also [15]. We note that in analogy with statistical mechanics, where temperature emerges as the inverse of the Lagrange multiplier in the derivation of the Boltzmann distribution and is considered as a statistical property of matter in bulk, $\beta$ can be viewed as an emergent quantity at a tissue level.

\subsection{Special case: Lemaître's law}

Lemaitre's law is the virial equation of state, originally proposed for two-dimensional foams, which relates two measures of disorder (thermodynamic variables), namely, the fraction of hexagons to the width of the polygon distribution, in cellular mosaics $[16,17,18,19]$. It can be obtained by maximizing the entropy, $S=-\sum_{n=3} p_{n} \ln p_{n}$, where $p_{n}$ is the probability of having an $n$-sided polygon, while considering the following information:

$$
\sum_{n=3} p_{n}=1, \quad \sum_{n=3} n p_{n}=6, \quad \sum_{n=3} f_{n} p_{n}=\text { const. },
$$

where the first relation concerns the normalization condition and the second is a consequence of Euler's relation concerning the topology of the structure, which assumes only three lines meet at the same vertex (as, in this paper, we are interested in Voronoi tessellations, this is always the case). The function $f_{n}$, in the last relation, depends on the geometry or the underlying dynamics of the cells (polygons). Lemaitre et al. [16, 17] originally considered $f_{n}=1 / n$, as an empirical observation made through measuring the areas of cells in twodimensional mosaics produced by hard discs moving on an air table. It has been shown that a wide range of two-dimensional cellular networks in nature, ranging from biology, such as epithelial cells, to physics, such as Ising model and amorphous graphene, obey Lemaitre's law [19, 20, 21, 22, 23]; although, at first sight, it seems that the choice of $f_{n}=1 / n$ is not applicable to a general setting. In fact, it was already mentioned in [17] that this particular form of $f_{n}$ 
cannot be valid for all cellular mosaics, for instance, it is incompatible with the well-known Lewis' law [24], which assumes a linearity in $n$; however, the authors of [17] speculated that the remarkable universality of Lemaitre's law suggests that the constraint on $f_{n}=1 / n$ has probably a deeper meaning than expected.

In the following, we derive Lemaitre's law as a special case of our formalism, explained in the beginning of Sec. 2. The main idea is to consider a general standardized discrete distribution for the calculation of the entropy; as a result of this, we can justify the particular choice of $f_{n}=1 / n$ in (5) and show the universality of Lemaittre's law. To this end, first we generalize the Lagrangian introduced in (3) as

$$
\begin{gathered}
L=S(X)-\lambda\left[\sum_{x \in \mathcal{X}} p(x)-1\right]-\beta\left[\sum_{x \in \mathcal{X}} p(x) S(Y \mid X=x)-\bar{S}\right] \\
-\mu\left[\sum_{x \in \mathcal{X}} p(x) N(Y \mid X=x)-\bar{N}\right],
\end{gathered}
$$

where we have assumed the following additional information: $N(Y \mid X)=$ $\sum_{x \in \mathcal{X}} p(x) N(Y \mid X=x)=\bar{N}$, which $N(Y \mid X)$ is the average number of cells in the microenvironment, and we have introduced the corresponding Lagrange multiplier as $\mu$. Now, by solving $\partial L / \partial p(x)=0$, we obtain:

$$
p(x)=\frac{e^{-\beta S(Y \mid X=x)-\mu N(Y \mid X=x)}}{Z},
$$

where $Z=\sum_{x \in \mathcal{X}} \exp [-\beta S(Y \mid X=x)-\mu N(Y \mid X=x)]$. We can simplify the notations in (7) and write:

$$
p_{n}=\frac{e^{-\beta S_{n}-\mu n}}{Z},
$$

where $p_{n}$ is the probability of having an $n$-sided polygon and $Z=\sum_{n=3} \exp \left[-\beta S_{n}-\right.$ $\mu n]$. Now, in order to calculate $S_{n}$, we consider a general standardized discrete distribution, which its density can be expanded as [25],

$$
g_{n}(x)=\frac{1}{\sqrt{2 \pi}} e^{-\frac{x^{2}}{2}}\left\{1+\frac{g_{1}}{\sqrt{n}}+\frac{g_{2}}{n}+\cdots\right\}
$$

where $g_{1}=\alpha_{1} H_{3}(x / \sqrt{2})$ and $g_{2}=\alpha_{2} H_{4}(x / \sqrt{2})+\alpha_{3} H_{6}(x / \sqrt{2})$, for some constants $\alpha_{1}, \alpha_{2}, \alpha_{3}$, and $H_{k}(\cdot)$ is the $k$ th Hermite polynomial. We note that, as $n \rightarrow \infty, g_{n}(x)$ approaches the standard normal distribution. Now that we have $g_{n}(x)$ at our disposal, we can calculate the differential entropy $S_{n}=-\int_{-\infty}^{\infty} d x g_{n}(x) \ln g_{n}(x)$; since $H_{3}(x)=8 x^{3}-12 x$ is an odd function of $x$, its integral vanishes, as a result, the first nonzero correction term is of the order $1 / n$. Thus, for a general standardized discrete distribution, we obtain:

$$
S_{n}=\frac{1}{2} \ln (2 \pi e)+\mathcal{O}\left(\frac{1}{n}\right),
$$


where the first term is the entropy of the standard normal. Now, by plugging (10) into (8), we arrive at

$$
p_{n}=\frac{e^{-\beta / n-\mu n}}{Z}
$$

where $Z=\sum_{n=3} \exp [-\beta / n-\mu n]$.

The variance, $\mu_{2}$, of the distribution $p_{n}$ in (11) reads as

$$
\mu_{2}=\left\langle(n-\langle n\rangle)^{2}\right\rangle=\sum_{n=3} p_{n}(n-6)^{2},
$$

where we have used Euler's relation. The second moment of $p_{n}$, i.e., $\mu_{2}$, demonstrates a deviation from the hexagonal configuration, thus it can be interpreted as a measure of topological disorder.

By exploiting (11) and (12), Lemaitre's law, as a relation between two measures of disorder, that is, $\mu_{2}$ and $p_{6}$, has been obtained as [16, 17, 18, 19, 23],

$$
\begin{aligned}
\mu_{2} p_{6}^{2}=1 /(2 \pi), & 0.34<p_{6}<0.66, \\
\mu_{2}+p_{6}=1, & 0.66<p_{6} \leq 1 .
\end{aligned}
$$

In the following, we present a simple and intuitive derivation of Equations (13) and (14). For (13) to hold, since we have assumed $p_{6}$ is large enough, we suppose $p_{n}$ in (11) is peaked at $n=6$, thus we can approximate it - near $n=6$ - by a normal distribution, $P_{n}$, centered at $n=6$, while ignoring the discreteness of $n$. We also allow $n$ to vary from $-\infty$ to $\infty$, which can be justified as only the values of $n$ close to the peak at $n=6$ have significant contributions, provided that $p_{6}$ is not too small. Thus, we have: $P_{n}=1 / \sqrt{2 \pi \mu_{2}} \exp [-(n-$ $\left.6)^{2} /\left(2 \mu_{2}\right)\right]$, which results in $\mu_{2} P_{6}^{2}=1 /(2 \pi)$. Now, for Equation (14) to hold, we need probabilities, $p_{n}$ 's, for $n \notin\{5,6,7\}$ to be negligible compared with $p_{n}$ 's for $n \in\{5,6,7\}$, as a result, in this region, the discreteness of $n$ cannot be neglected as only three values contribute. Since $\langle n\rangle=6$, this implies that $p_{n}$, thus $h(n) \equiv-\beta / n-\mu n$, should sharply peak at $n=6$, which leads to $\mu_{2}+p_{6} \rightarrow 1$ as $p_{6} \rightarrow 1$. We note that, although in (6), we assumed information about seemingly unrelated quantities of $S(Y \mid X)$ and $N(Y \mid X)$, represented in terms of their corresponding Lagrange multipliers, $\beta$ and $\mu$, respectively, however, the peakedness of $p_{n}$ at $n=6$ gives us a relation between $\beta$ and $\mu$; since $h^{\prime}(6)=\beta / 6^{2}-\mu=0$, thus we have: $\beta=36 \mu$. Also, in order $h^{\prime \prime}(6)=$ $-2 \beta / 6^{3}<0, \beta$ must be positive, which establishes a necessary condition in order to have an ordered crystalline structure, that is, to have an abundance of hexagons. If $\beta<0$, then $h(n)$ is minimized for $n=6$, which implies the corresponding minimization of $p_{n}$ for $n=6$. Such a configuration which is characterized by the lack of hexagons, indicates an amorphous structure, that is, a highly disordered pattern. As an illustration, in Figure 1, we have shown the discrete plot of $\left\{\left(n, p_{n}\right): n \in\{3, \ldots, 10\}\right\}$ for $\mu=-0.1$ and $\beta=-3.6$, where the percentage of hexagons is around $11 \%$. In the biology context, $\beta<0$ could imply the early stage of the organism development, which the system has not yet reached the organized state, and the disordered and ordered phases could be 


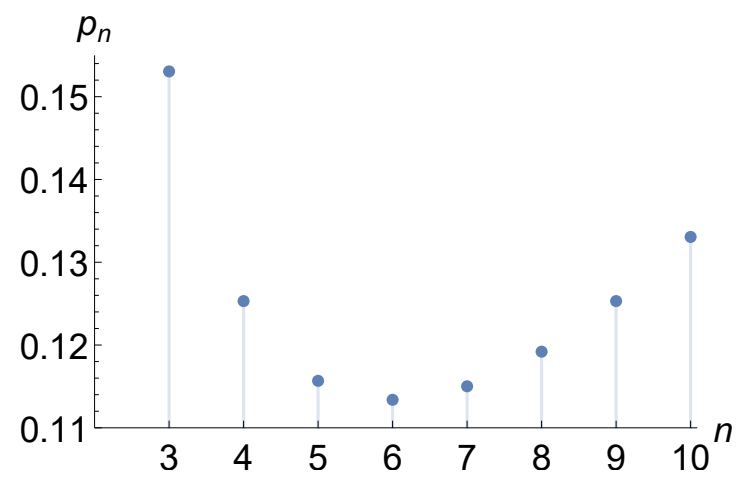

Figure 1: The discrete plot of $\left\{\left(n, p_{n}\right): n \in\{3, \ldots, 10\}\right\}$ for $p_{n}$ in (11), where $\mu=-0.1$ and $\beta=-3.6$. The figure shows a low percentage of hexagons $(\approx 11 \%)$, for which $\beta$ is negative.

linked by a phase transition; or, it could imply malfunctioning of the organism.

To obtain the regions of validity of the derived equations in the previous paragraph, we have analyzed (11), (13), and (14) numerically, and the results are presented in Figure 2. The left panel illustrates $\mu_{2}$ as a function of $p_{6}$, where the red points are obtained directly from (11), subjected to the constraint $\langle n\rangle=6$, and the blue and green curves correspond to (13) and (14), respectively. Our numerical analysis suggests that the known lower bound of (13), reported in the literature, can be relaxed to 0.20 , that is,

$$
\mu_{2} p_{6}^{2}=1 /(2 \pi), \quad 0.20 \leq p_{6}<0.66 .
$$

In the right panel of Figure 2, we have shown $\beta$ as a function of $\mu$, where we have compared the analytical result of $\beta=36 \mu$, as a blue curve, with the values obtained from (11), subjected to the constraint $\langle n\rangle=6$.

We conclude by noting that, the arguments provided in this section can be reformulated with the corresponding modified results, if other $p_{n}$ 's, rather than $p_{6}$, have the largest contributions; in Sec. 3 , such systems are studied.

\section{Spatial distributions of the cone photorecep- tors in the retinas of vertebrates}

In this section, we want to examine the results obtained in the previous section, namely, (4), (14), and (15), by applying them to the retinal cone cell mosaics of rodent, dog, monkey, human, fish, and bird. We demonstrate that the application of the principle of maximum entropy leads to the introduction of a new parameter, which is conserved among different species of vertebrates; we call this coarse-grained parameter as collective sensing. In Sec. 3.1, we elaborate on 

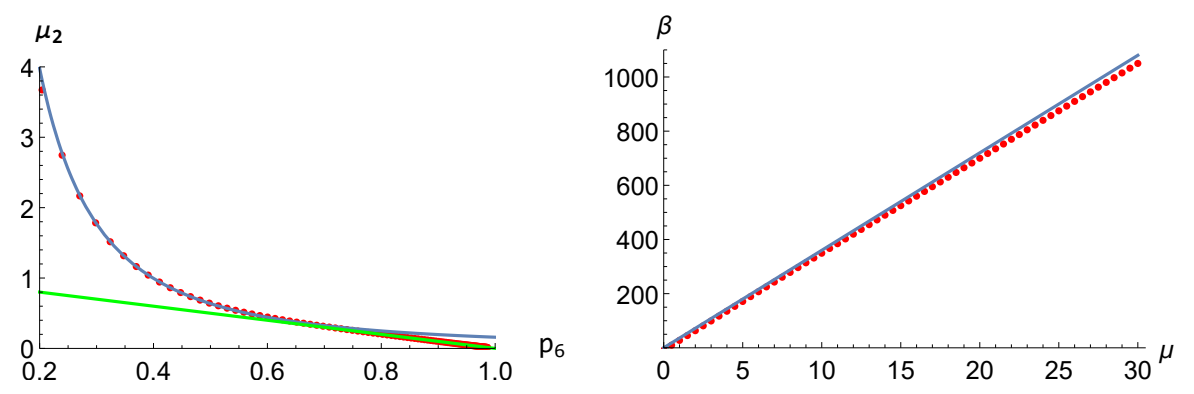

Figure 2: In the left panel, the blue and green curves correspond to (13) and (14), respectively; the red points are obtained directly from (11), subjected to the constraint $\langle n\rangle=6$. This plot suggests that the known lower bound of (13) can be relaxed to 0.20 . The comparison between the analytical result of $\beta=36 \mu$ (blue curve) and the $\beta$ 's and $\mu$ 's obtained from (11) is depicted in the right panel.

the details of our calculations in the case of the human cone cells; other species are presented in Sec. 3.2.

\subsection{Spatial distributions of the human cone photorecep- tors}

Human color vision is mediated by three types of cone cells, which are sensitive to (blue) short- (S), (green) medium- (M), and (red) long- (L) wavelength light. The spatial distributions of these cells, in a living human eye, are shown in Figure 3. The image in the top-left corner, is the first image of the spatial arrangement of the living human cones, reported in [26].

Our first goal, here, is to predict the occurrence probabilities of the cone cells, i.e., the S, M, and L cones, in the retinal field of the human eye presented in Figure 3. To this end, we exploit (4) and rewrite it as

$$
p_{i}=\frac{e^{-\beta S_{i}}}{\sum_{j} e^{-\beta S_{j}}}=\frac{e^{-\beta S_{i}}}{e^{-\beta S_{b}}+e^{-\beta S_{g}}+e^{-\beta S_{r}}},
$$

where $p_{i}$ is the probability of observing the $i$ th color, i.e., the $i$ th cone subtype, and $b, g$, and $r$ stand for the blue, green, and red cones, respectively. For the sake of simplicity, we have considered the following two assumptions: in each cone subtype, we consider cells to be indistinguishable and we assume no explicit dependence of cones of different types on each other, thus, for instance, the microenvironment of a blue cone consists of other blues. The latter can be justified as this dependence is already encoded in the arrangement of the cells with respect to each other, in the whole pattern.

Now, we need to calculate $S_{b}, S_{g}$, and $S_{r}$, which in turn, first we need to consider some density functions to begin with. Our choice is to construct the 


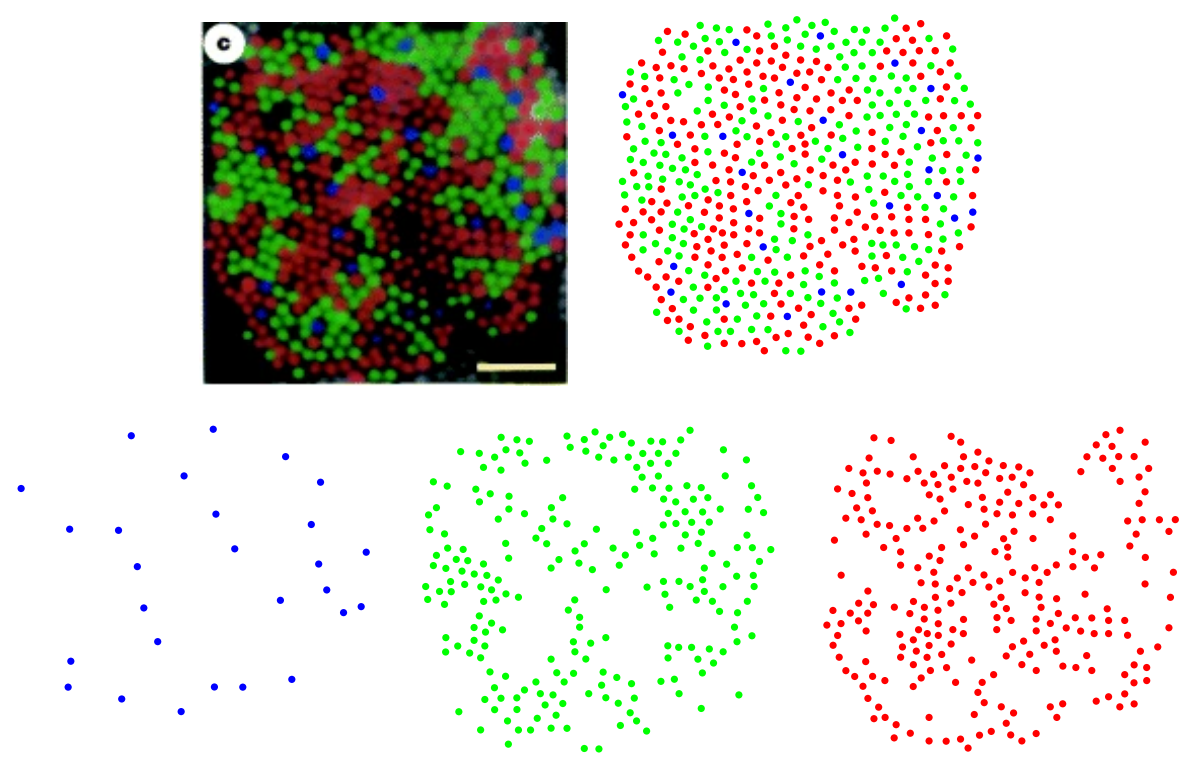

Figure 3: The spatial distributions of the cone photoreceptors in a living human nasal retina, at one degree of eccentricity. The image, in the top-left corner, is adapted from [26], where the scale bar $=5 \mu \mathrm{m}$. The figures in the bottom row, from left to right, illustrate the short-, medium-, and long-, wavelength-sensitive cones, separately. 


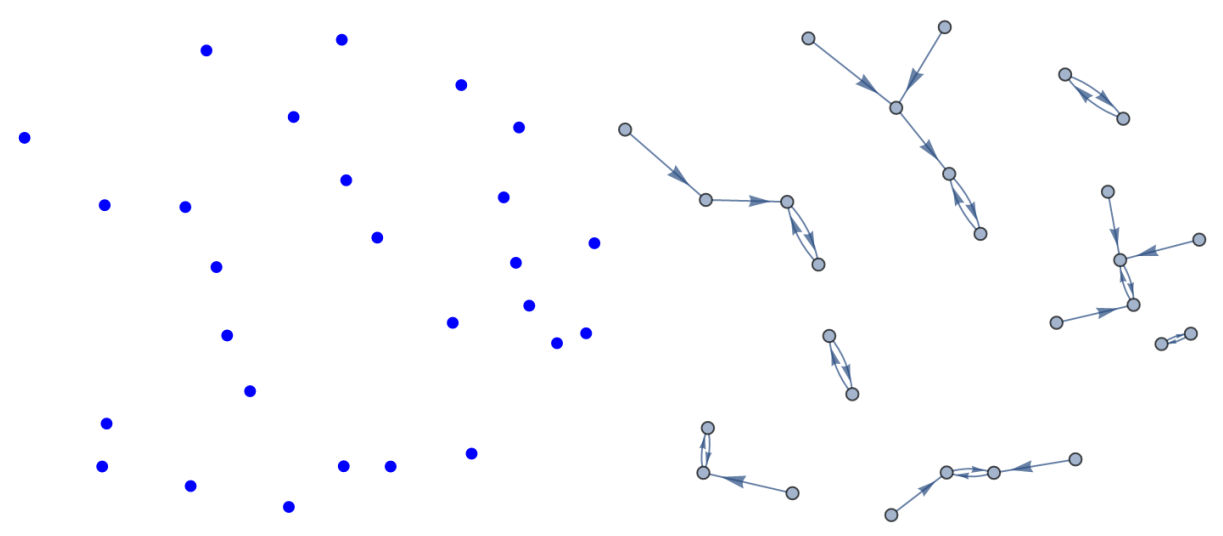

Figure 4: Searching for the nearest neighbors, in the case of the blue cone photoreceptors, in a living human retina.
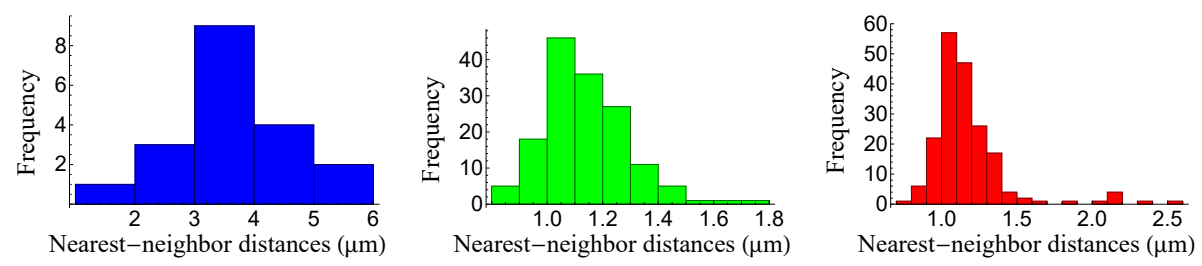

Figure 5: The nearest-neighbor-distance distributions of the cone photoreceptors in a living human retina. The values of the mean and standard deviation (in micrometers), for each distribution, read as: $\mu_{b}=3.572, \sigma_{b}=1.020, \mu_{g}=1.188$, $\sigma_{g}=0.300, \mu_{r}=1.172$, and $\sigma_{r}=0.257$.

nearest-neighbor-distance (NND) distribution for each cone subtype and then to calculate its differential entropy. The rationale behind our choice is that, the methods based on the concept of the nearest-neighbor distance have been extensively used to quantify cone mosaics, see for example [11], which turns out to be a simple, but powerful, concept to analyze spatial patterns. As an example, in Figure 4, we have shown searching for the nearest neighbors in the case of the blue cones.

The NND distribution for each cone subtype is presented in Figure 5, which demonstrates that the nearest-neighbor distances, in each case, follow an approximately normal distribution. We note that, in order to obtain the optimal bin widths of the histograms, we have used a data-based procedure proposed by M. P. Wand [27], to its first-order approximation, which is called the one-stage rule (the zeroth-order approximation, i.e., the zero-stage rule, of the method reproduces Scott's rule of binning [28]). Now, we can construct the probability density function, $P_{i}(x)$, for each histogram in Figure 5 , such that $\int d x P_{i}(x)=1$; then, the differential entropy can be calculated according to: 

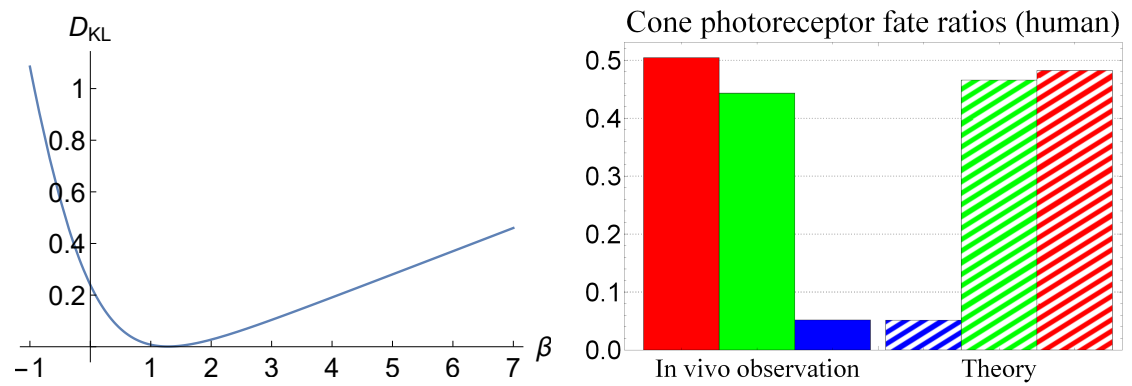

Figure 6: The Kullback-Leibler divergence is depicted as a function of $\beta$ in the left panel, where it has the global minimum of 0.001 at $\beta=1.284$. The right panel shows the comparison between the in vivo observed cone photoreceptor percentages, in the human retina, and the predictions of the theory, that is, (16), for $\beta=1.284$.

$S_{i}=-\int d x P_{i}(x) \ln P_{i}(x)$. We obtain the entropies as

$$
S_{b}=1.365, \quad S_{g}=-0.350, \quad S_{r}=-0.378 .
$$

From the image in the top-left corner of Figure 3, we can determine the occurrence probabilities of the S, M, and L cones, which are the percentages of these cells in the retinal field. Now, we need to find the appropriate $\beta$ in (16), which results in predicting the observed values of the probabilities. To this end, we employ the Kullback-Leibler divergence, that is, $D_{\mathrm{KL}}=\sum_{i} q_{i} \ln \left(q_{i} / p_{i}\right)$, where $q_{i}$ and $p_{i}$ correspond to the in vivo observed values of the cone cell probabilities and the predictions of the theory, i.e., (16), respectively. The left panel of Figure 6 illustrates the Kullback-Leibler divergence as a function of $\beta$, with the global minimum of 0.001 at $\beta=1.284$. The observed cone percentages, in a living human retina, are compared to the predictions of the theory for $\beta=1.284$, in the right panel of Figure 6; this figure proves our assertion made in Sec. 2, that is, receptors with lower microenvironmental entropy have a higher probability of occurrence. Since at the heart of this approach is the arrangement of the cells with respect to each other, we call the parameter $\beta$ as collective sensing.

Our second goal, here, is to examine the modified Lemaître's law in the case of the human eye. In order to partition the retinal fields of Figure 3 into polygons, we construct the corresponding Voronoi tessellations. In this structure, each Voronoi polygon is generated by a cone cell in such a way that all points in a given polygon are closer to its generating cone cell than to any other [29]. In the top row of Figure 7, we have depicted the Voronoi tessellations of the spatial arrangements of the S, M, and L cones in the human retina and in the bottom, the Voronoi tessellation of the whole pattern of the cones is presented. The fractions of the $n$-sided bounded polygons are reported in the figure caption. If we assume a high value of $p_{6}$ indicates the regularity of the corresponding cone mosaic, Figure 7 demonstrates that the spatial arrangement of the $\mathrm{S}$ cones is more random than those of the $\mathrm{M}$ and $\mathrm{L}$ cones, where for the 

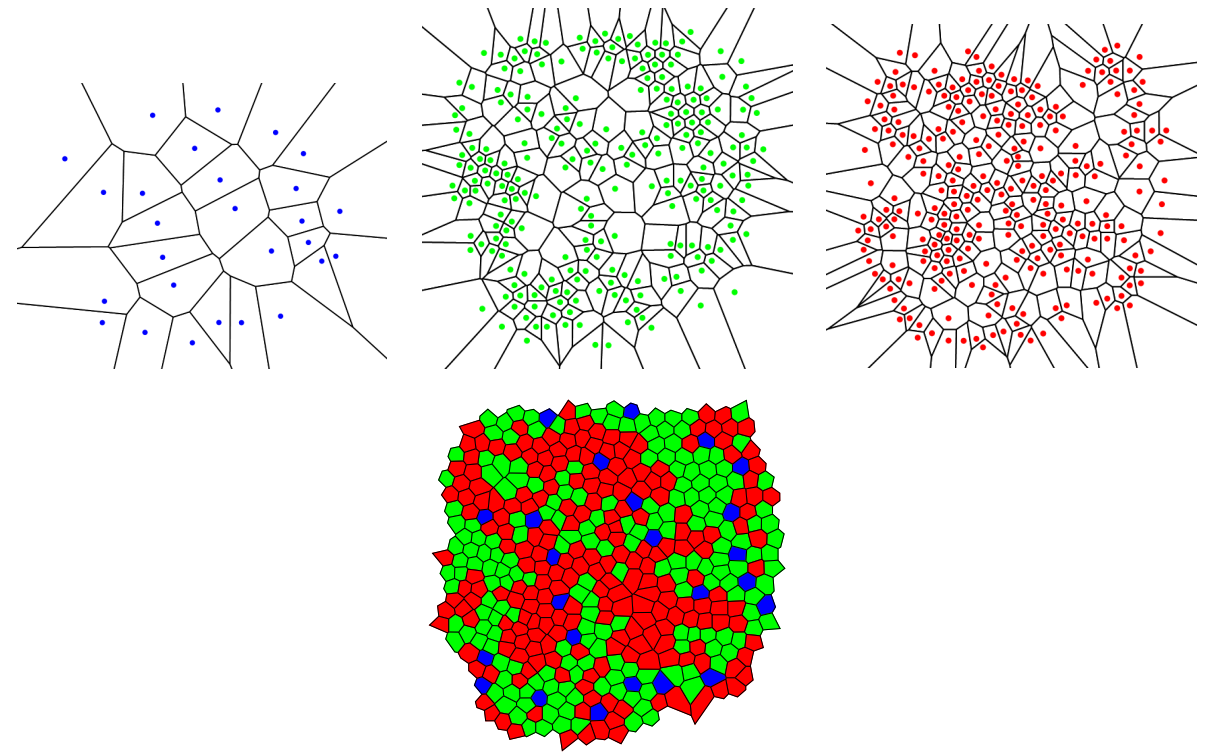

Figure 7: In the top row, we have shown the Voronoi tessellations regarding the three cone photoreceptor subtypes in a living human retina. The fractions of the $n$-sided bounded polygons, $p_{n}$ 's, in each case, are: $p_{4}^{b}=0.286, p_{5}^{b}=0.286$, $p_{6}^{b}=0.143, p_{7}^{b}=0.214$, and $p_{8}^{b}=0.071 ; p_{3}^{g}=0.010, p_{4}^{g}=0.089, p_{5}^{g}=0.300$, $p_{6}^{g}=0.360, p_{7}^{g}=0.153, p_{8}^{g}=0.069$, and $p_{9}^{g}=0.020 ; p_{4}^{r}=0.077, p_{5}^{r}=0.300$, $p_{6}^{r}=0.378, p_{7}^{r}=0.184, p_{8}^{r}=0.030$, and $p_{9}^{r}=0.030$. The Voronoi tessellation of the whole retinal field is illustrated in the bottom, with the fractions of the $n$-sided polygons as: $p_{4}=0.012, p_{5}=0.171, p_{6}=0.718, p_{7}=0.086$, and $p_{8}=0.012$. 

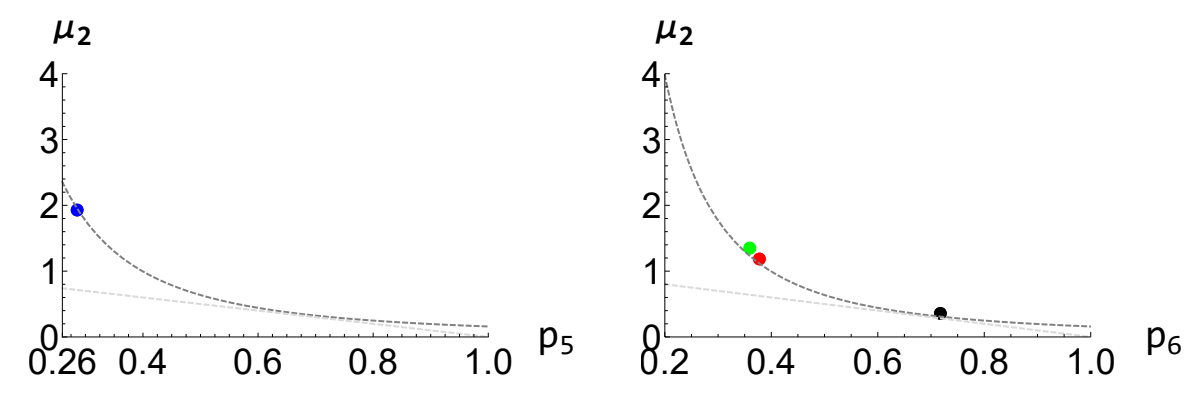

Figure 8: The blue, green, red, and black points depict the experimental values of $\left(p_{i}, \mu_{2}\right), i=5,6$, regarding the $\mathrm{S}, \mathrm{M}, \mathrm{L}$, and the whole pattern of the cones, respectively. The theoretical result, i.e., Lemaitre's law, is represented as the light gray curve and the dark gray, dashed one, which correspond to (14) and (15), respectively.

blue cones, we have: $p_{6}^{b}=0.143$, while $p_{6}^{g}=0.360$ and $p_{6}^{r}=0.378$, for the greens and reds, respectively. This finding agrees with [26]. We also note that, as is shown in the bottom of Figure 7, in contrast to the cone subtypes, the whole spatial arrangement of the human cones is highly ordered, with $p_{6}=0.718$.

Figure 8 shows Lemaitre's law as is applied to the human cone photoreceptors. Although, the blue cones have a low $p_{6}$, however, there is a significant contribution of pentagons in the Voronoi tessellation. As explained at the end of Sec. 2, our arguments leading to Lemaitre's law can be reformulated with the corresponding modified results, if other polygons have the largest contributions, which in this case, it is $p_{5}$. In the left panel of the figure, the experimental value of $\left(p_{5}, \mu_{2}\right)$ regarding the $\mathrm{S}$ cone, is represented in blue, and the light gray curve and the dark gray, dashed one correspond to the modified versions of (14) and (15) for $p_{5}$, respectively. The cases of the M (green), L (red), and the entire pattern of the cones (black), are shown in the right panel. In all cases, the agreements between Lemaitre's law and the observed values of $\left(p_{i}, \mu_{2}\right), i=5,6$, are quite good.

As another illustration, in Figure 9, we have investigated the behavior of the human cones with respect to Lemaître's law, at six different retinal locations in a living human eye, namely, two, four, six, eight, ten, and twelve degrees of retinal eccentricities, temporal to the fovea.

\subsection{Spatial distributions of the vertebrate cone photore- ceptors: from rodent to bird}

In this subsection, we apply the procedures explained in Sec. 3.1 to various vertebrate species, namely, rodent, dog, monkey, human, fish, and bird. The rodent and dog are dichromats, the monkey, like the human, is trichromat, and the fish and bird are tetrachromats, which in the case of the bird, there is also a significant number of double cones. The results are summarized in Figures 10, 

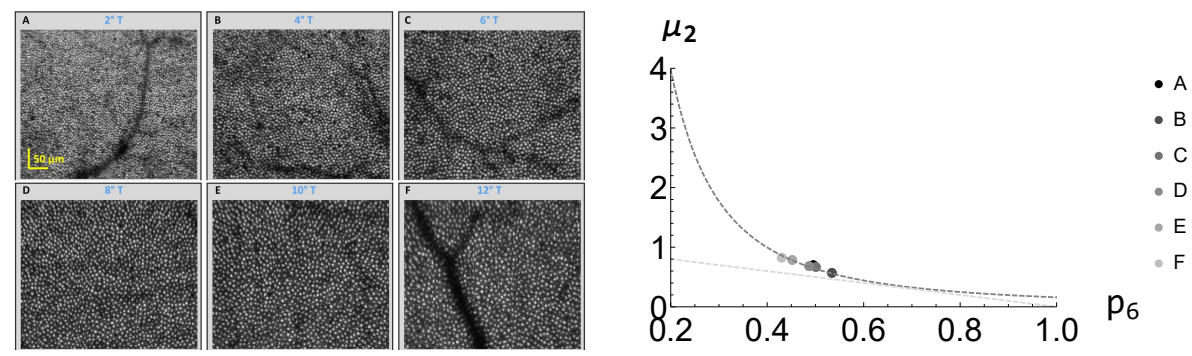

Figure 9: The left panel, adapted from [30], demonstrates the spatial distributions of the cone photoreceptors in the retina of a living human eye at a range of retinal eccentricities. In the right panel, we have shown the behavior of the cones - the whole pattern in each case - with respect to Lemaitre's law.

$11,12,13,14$, and 15. Although, the cone mosaics of these diverse species are significantly different from each other, the values of the parameter $\beta$, in all cases, are in the same order, where $1<\beta<2$. In the next section, by using statistical analyses and the fact that the NND distributions of the cone subtypes, in the mentioned species, are peaked distributions and thus can be approximated by Gaussians, we estimate the value of $\beta$ in a general case.

\section{Discussion}

We are in a position to address the issue raised in the beginning of Sec. 2: although, we lacked the knowledge of the numerical value $\bar{S}$ of the microenvironmental entropy, however, we considered it as a crucial information about the system and represented it in terms of the Lagrange multiplier $\beta$. In this section, we want to estimate the value of $\bar{S}$, which leads to the estimation of $\beta$, by considering an additional information obtained in Sec. 3, that is, the fact that the distribution of the nearest-neighbor distances of a given cone subtype can be approximated by a Gaussian distribution.

First, we note that, in the case of a normal distribution where $S_{i}=(1 / 2) \ln \left(2 \pi e \sigma_{i}^{2}\right)$, (4) becomes:

$$
p_{i}=\frac{\sigma_{i}^{-\beta}}{Z}
$$

where $i$ denotes colors and $Z=\sum_{j} \sigma_{j}^{-\beta}$. We also assume the nearest-neighbor distances as random variables $X_{i}$ 's, where $X_{i} \sim \mathcal{N}\left(\mu_{i}, \sigma_{i}^{2}\right)$. Now, in order to estimate $\bar{S}$, first we define the random variable $W$ as

$$
W=\sum_{i} \pi_{i} X_{i}
$$

where $\pi_{i}$ is the weight of the contribution of each cone subtype and $\sum_{i} \pi_{i}=1$. Since $W$ has a normal distribution, its entropy is related to its variance $\sigma^{2}$, as 

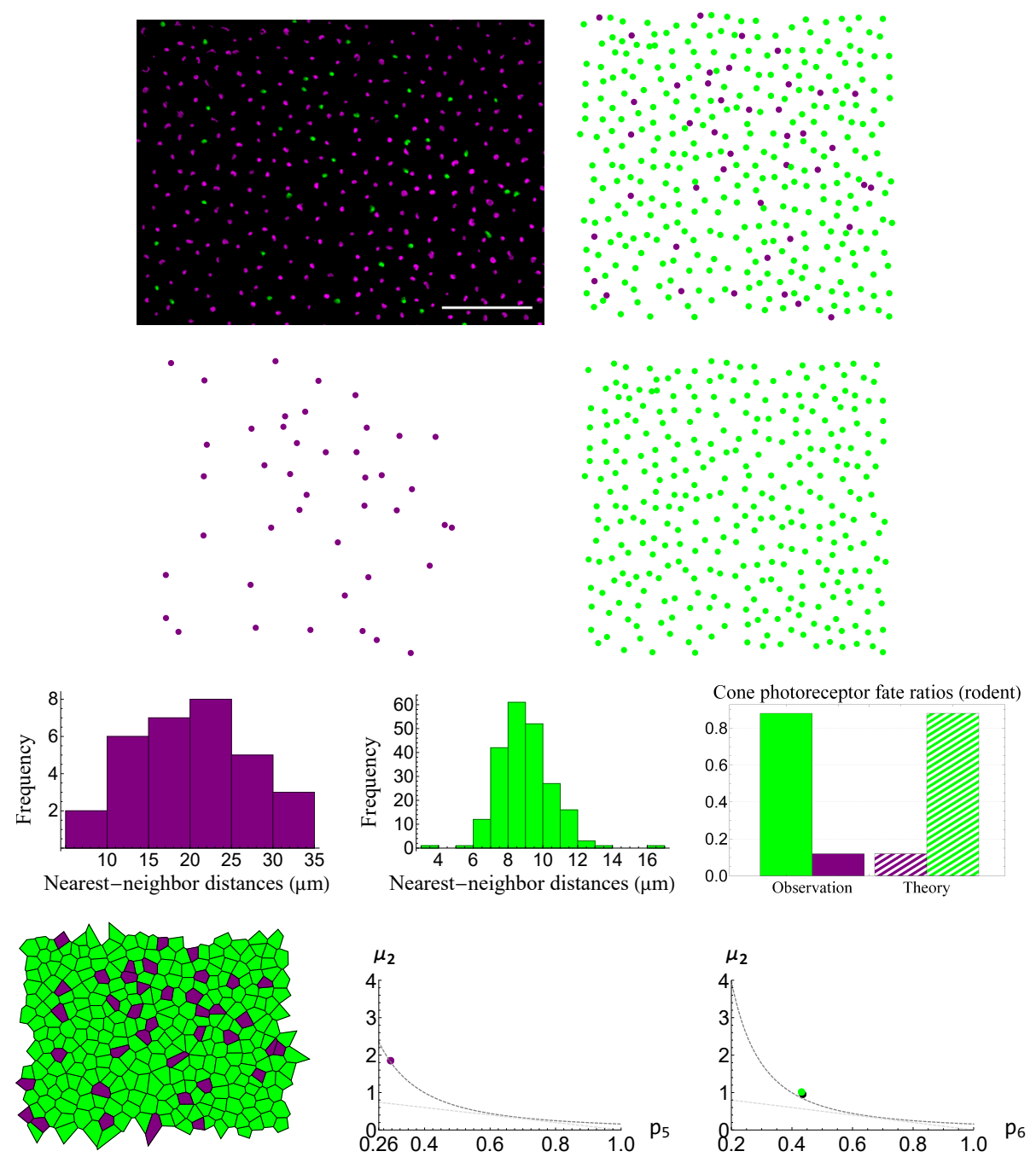

Figure 10: The image in the top-left corner (scale bar $=50 \mu \mathrm{m}$ ), adapted from [31], illustrates the spatial distribution of the cone photoreceptors in the dorsal mid-peripheral retina of a diurnal rodent, called the agouti. In this image, the S-cone opsin is represented as green and the L-cone opsin as purple; next to it, in the digitized image, we have reversed the colors. The nearest-neighbor-distance distributions have the entropies of $S_{v}=3.310$ and $S_{g}=1.787$; next to them, we have shown the comparison between the experimental observations and the predictions of the theory, evaluated at $\beta=1.310$ (the global minimum of the Kullback-Leibler divergence). The Voronoi tessellation of the retinal field and the behavior of the cones with respect to Lemaitre's law are illustrated in the last row, where the purple, green, and black points correspond to the S, L, and the whole pattern of the cones, respectively. 

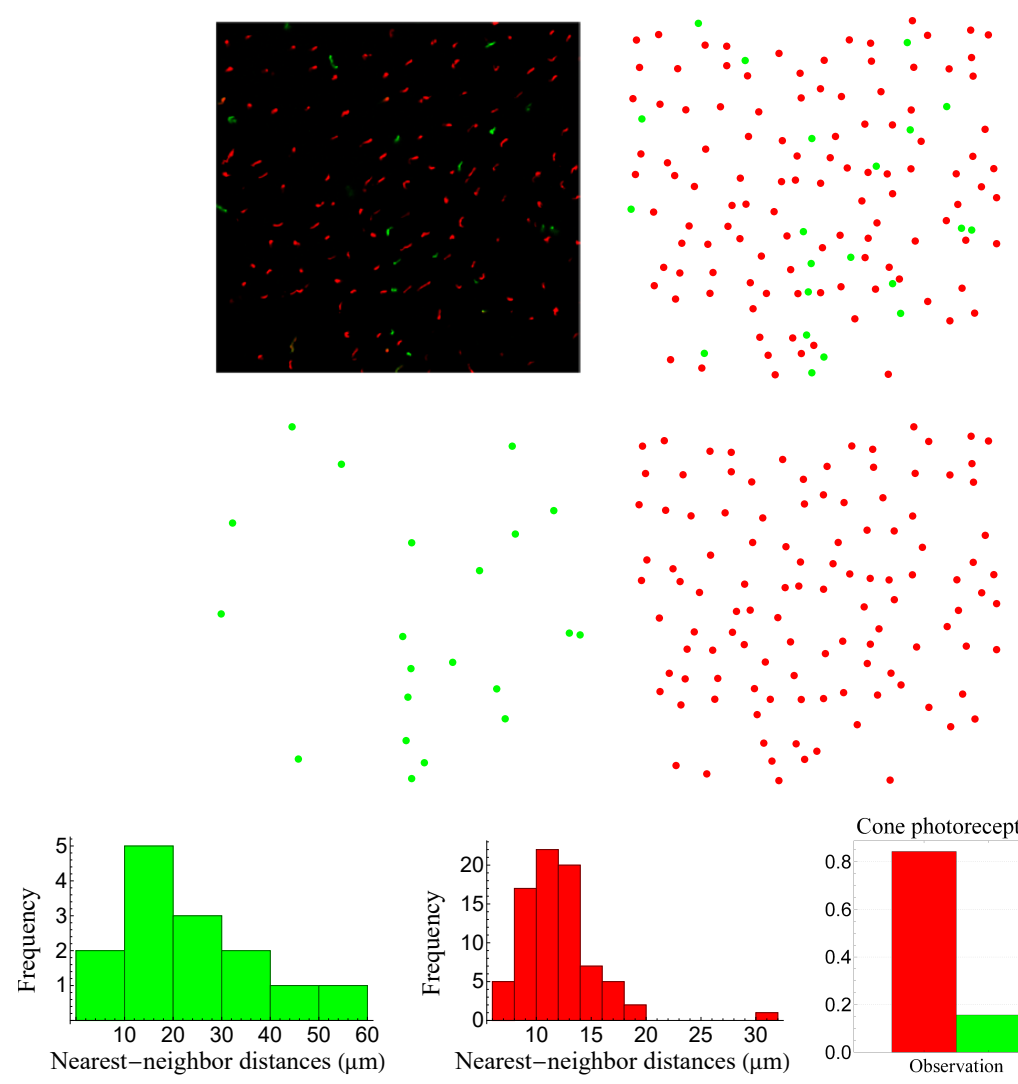

Cone photoreceptor fate ratios (dog)
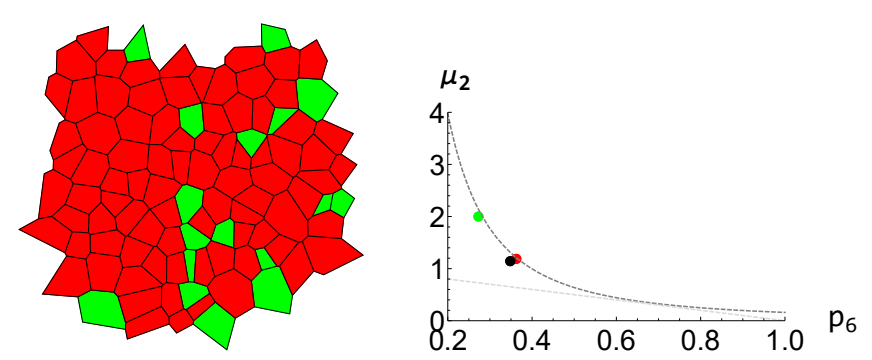

Figure 11: The image in the top-left corner, adapted from [32], shows the spatial distribution of the cone photoreceptors in the inferior peripheral retina of a dog; the $\mathrm{S}$-cone opsin is represented as green and the L/M-cone opsin as red. The entropies of the nearest-neighbor-distance distributions read as: $S_{g}=3.933$ and $S_{r}=2.440$. The comparison between the experiment and the predictions of the theory, as depicted in the third row, is evaluated at the global minimum of the Kullback-Leibler divergence, which occurs at $\beta=1.127$. The behavior of the cones with respect to Lemaitre's law is shown in the last row, where the green, red, and black points correspond to the experimental values of $\left(p_{6}, \mu_{2}\right)$ in the cases of the $\mathrm{S}, \mathrm{L} / \mathrm{M}$, and the whole pattern of the cones, respectively. 

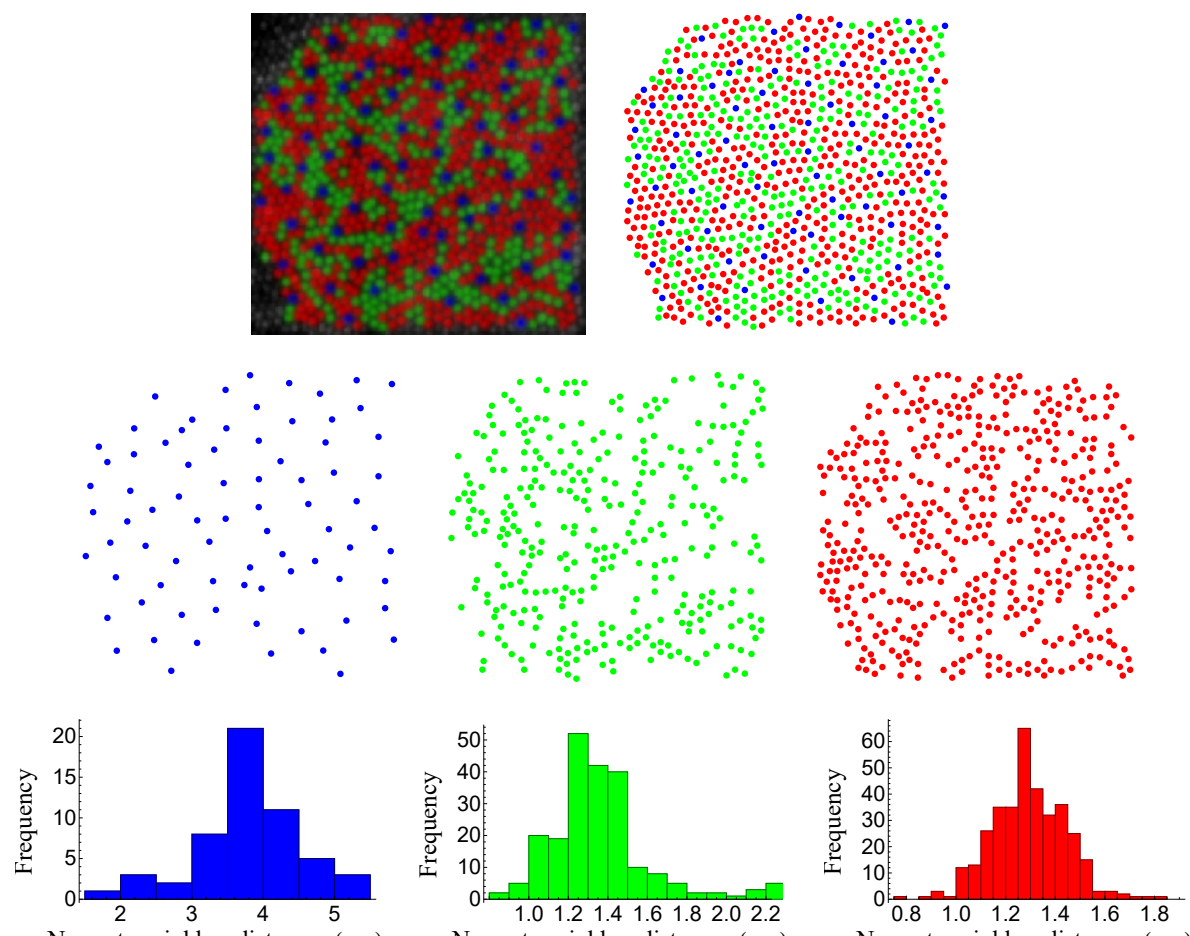

Nearest-neighbor distances $(\mu \mathrm{m})$

Nearest-neighbor distances $(\mu \mathrm{m})$

Nearest-neighbor distances $(\mu \mathrm{m})$
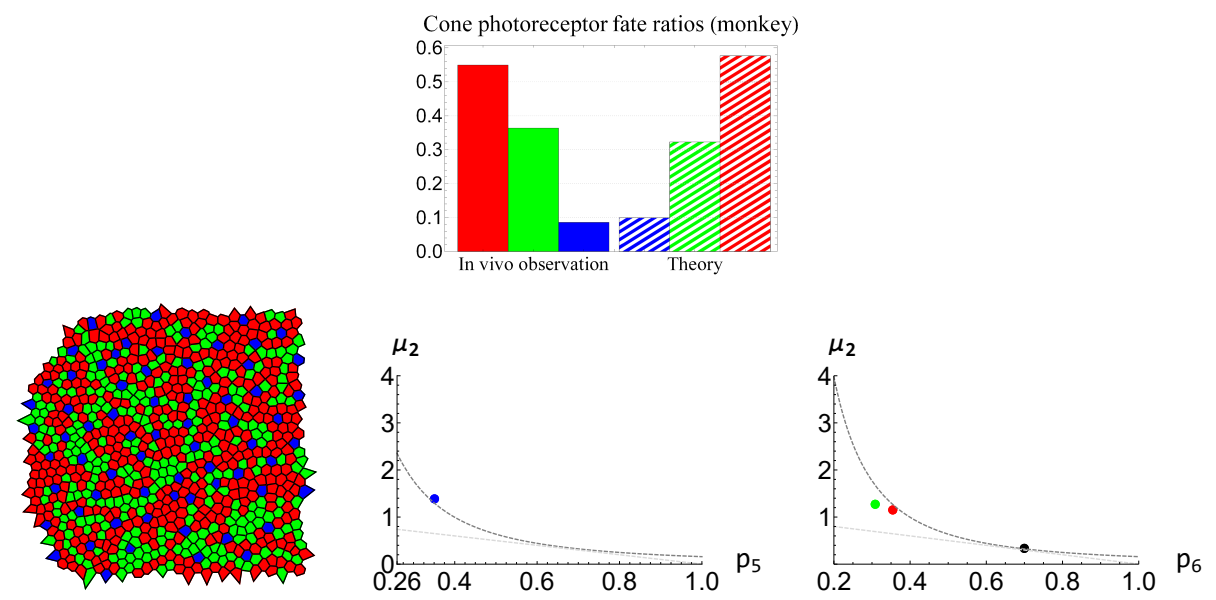

Figure 12: The image in the top-left corner, which shows the spatial distribution of the cone photoreceptors in the nasal retina of a monkey (macaque), is provided by A. Roorda [33]. The entropies of the nearest-neighbor-distance distributions read as: $S_{b}=1.019, S_{g}=0.018$, and $S_{r}=-0.476$. The predictions of the theory, illustrated in the fourth row, are evaluated at the global minimum of the Kullback-Leibler divergence, which occurs at $\beta=1.174$. 

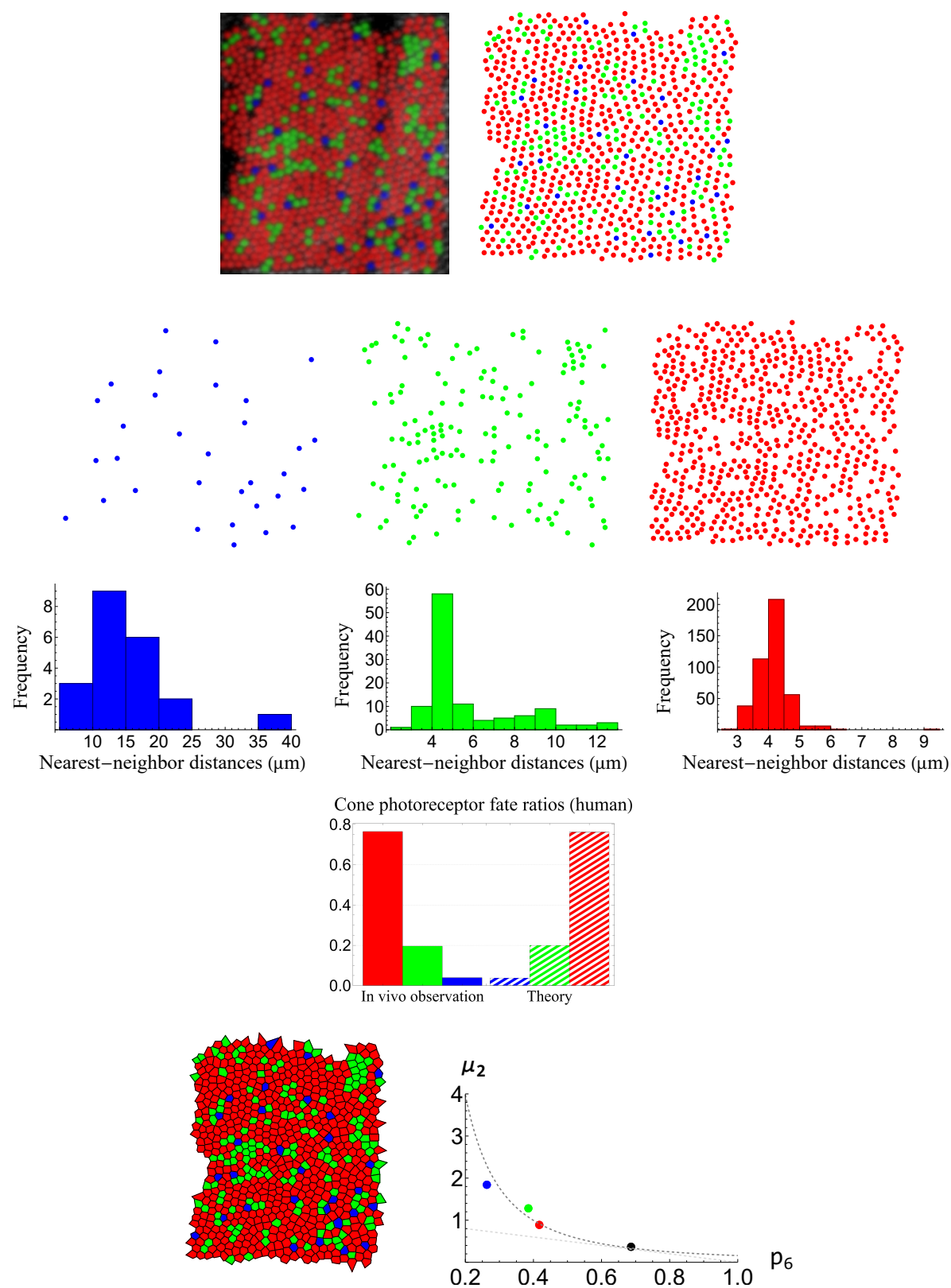

Figure 13: The image in the top-left corner, provided by A. Roorda [33], illustrates the spatial distribution of the cone photoreceptors in the temporal retina of a human. The entropies of the nearest-neighbor-distance distributions are: $S_{b}=2.977, S_{g}=1.691$, and $S_{r}=0.651 ;$ and, $\beta=1.291$. 
bioRxiv preprint doi: https://doi.org/10.1101/2022.01.09.475540; this version posted January 19,2022 . The copyright holder for this preprint (which was not certified by peer review) is the author/funder, who has granted bioRxiv a license to display the preprint in perpetuity. It is made available under aCC-BY-NC-ND 4.0 International license.

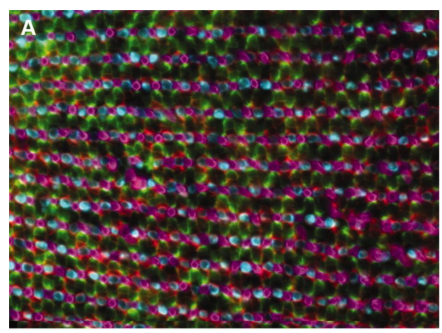

ระ)

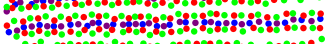

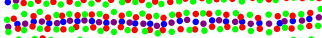
แใน ใน 78.

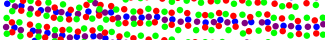
\$ร马 \%8 ระ8?

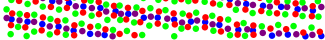

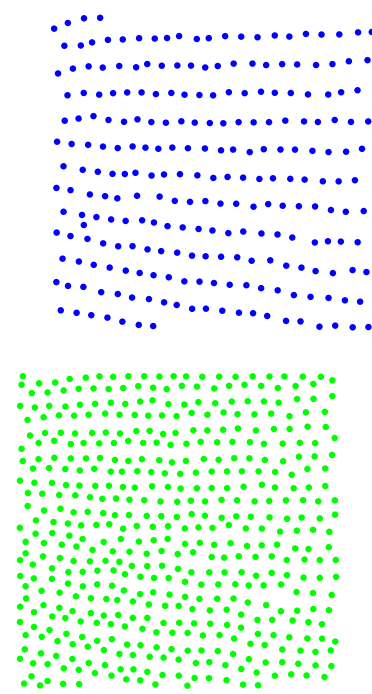

$\because \ldots \ldots \ldots \ldots \ldots \ldots$

$\therefore \therefore: \because: \because: \because \because \because \because \because \because::$ :

................

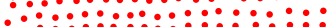

..................

(n..................

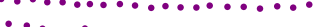

…............

$\ldots$

$\because \ldots \ldots \ldots . \cdots$

$\bullet \ldots \ldots \ldots \ldots \ldots, \ldots$

$\cdots \ldots \ldots \ldots \ldots$

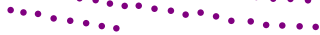

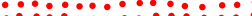

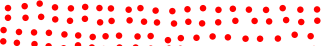

$\therefore:::::::::::::::::$

$\therefore::::::::::::$

$\because: \because:::::::::::$

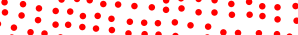

:: : : : :::::: :

$\therefore: \because: \because: \because: \because: \because: \because::$ : $: \because$
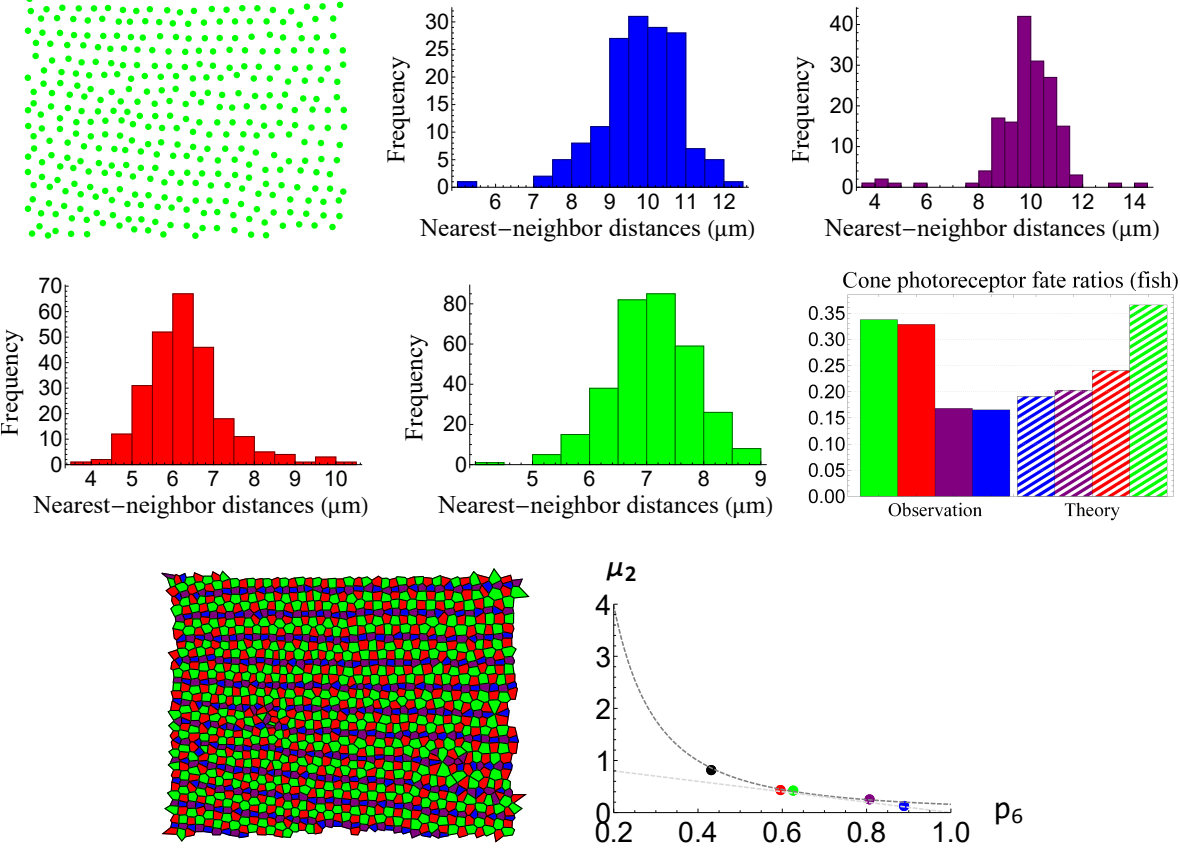

Figure 14: The image in the top-left corner, adapted from [34], shows the spatial distribution of the cone photoreceptors in the retina of the zebrafish. The corresponding entropies are: $S_{b}=1.471, S_{U V}=1.440, S_{r}=1.350$, and $S_{g}=1.128$; and, $\beta=1.894$. 
bioRxiv preprint doi: https://doi.org/10.1101/2022.01.09.475540; this version posted January 19,2022 . The copyright holder for this preprint (which was not certified by peer review) is the author/funder, who has granted bioRxiv a license to display the preprint in perpetuity. It is made available under aCC-BY-NC-ND 4.0 International license.
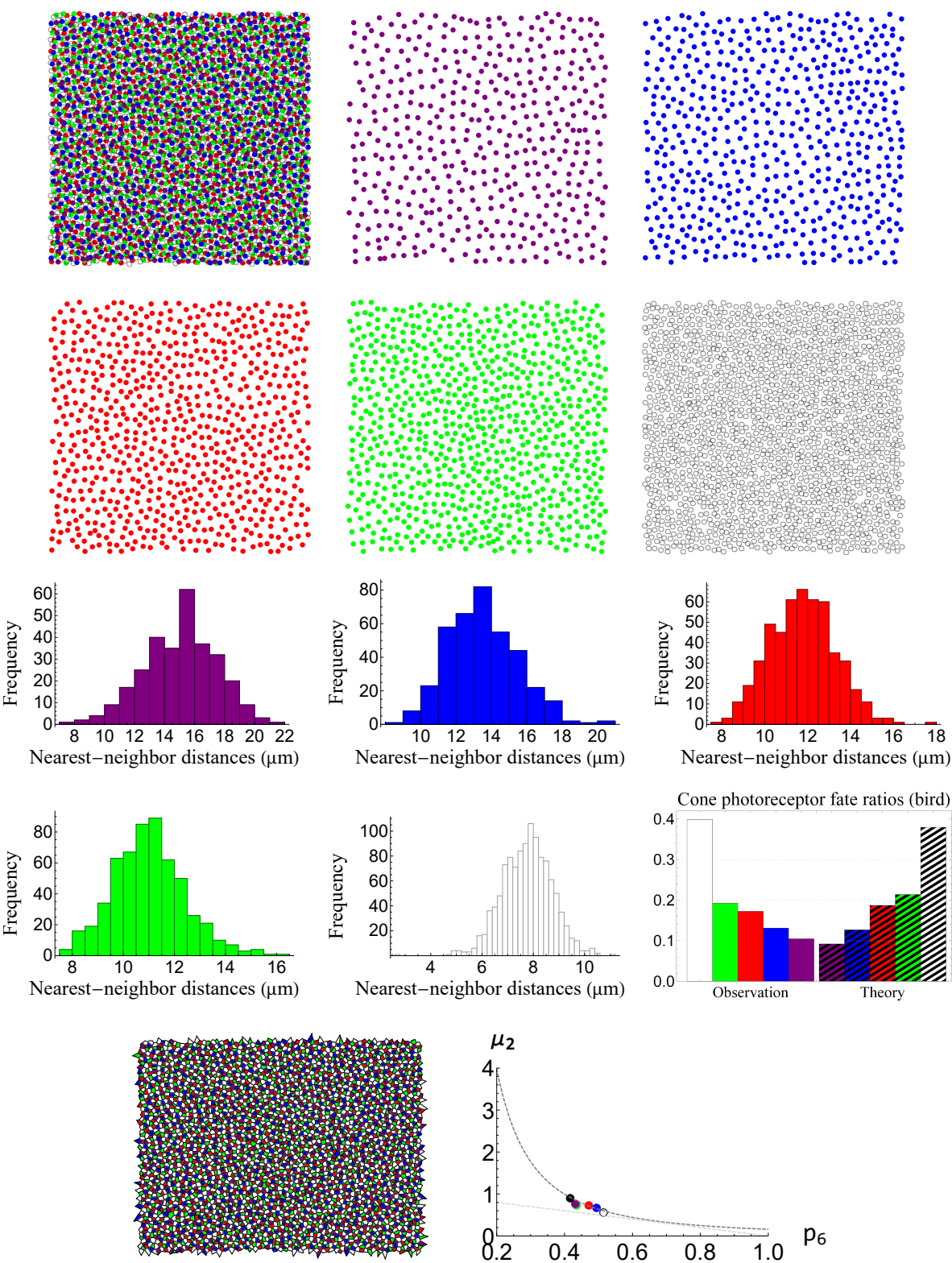

Figure 15: The digitized image of the spatial distribution of the cone photoreceptors in the dorsal nasal retina of the chicken, shown in the top-left corner, is constructed from the data reported in [35]; the double cones are represented as white. The corresponding entropies read as: $S_{v}=2.291, S_{b}=2.081, S_{r}=1.826$, $S_{g}=1.739$, and $S_{d}=1.364 ;$ and, $\beta=1.527$. 
$(1 / 2) \ln \sigma^{2}+$ const.; thus, in principle, we can obtain the lower and upper bounds of the entropy, by minimizing and maximizing the variance of $W$, respectively. In the following, we investigate these two extreme cases.

For the variance of $W$, we have: $\sigma^{2}=\sum_{i} \pi_{i}^{2} \sigma_{i}^{2}$. By the method of Lagrange multipliers, we can minimize $\sigma^{2}$, subjected to the constraint $\sum_{i} \pi_{i}=1$; it turns out that for $\pi_{i} \propto \sigma_{i}^{-2}, \sigma^{2}$ is minimized, which implies the minimization of the entropy of $W$. By comparing this $\pi_{i}$ with (18), we establish the upper bound of $\beta$ as 2 . We note that the two species of fish and bird, studied in the previous section, which their cone mosaics are more ordered - in the former highly regular and in the latter semi-random [13] — thus possessing lower entropies, their $\beta$ 's are closer to 2 than the other species.

Now, in order to obtain the lower bound of $\beta$, we should maximize the entropy of $W$, which implies the maximization of $\sigma^{2}$, subjected to $\sum_{i} \pi_{i}=1$. This happens by letting the $\pi_{i}$ corresponding to the largest $\sigma_{i}$ to be 1 and all the other $\pi_{i}$ 's vanish. This scenario is not desirable, as the contributions of different colors vanish. In order to obtain an acceptable maximum value of $\sigma^{2}$, we assume the uncertainties associated with the random variables $\pi_{i} X_{i}$ are equal, that is, we equalize the variances of $\pi_{i} X_{i}$ by considering $\pi_{i} \propto \sigma_{i}^{-1}$, which results in: $\sigma^{2}=C^{2} \sum_{i}=C^{2} N$, where $C$ is the proportionality constant, i.e., $\pi_{i}=C \sigma_{i}^{-1}$, and $N$ is the number of colors. Now, by comparing this $\pi_{i}$ with (18), we establish the lower bound of $\beta$ as 1 .

Hence, for the retinas of vertebrates, under the assumption that the NND distributions are Gaussians, we always have: $1<\beta<2$, as demonstrated explicitly for several species in Sec. 3 .

\section{Concluding remarks}

In summary, we have applied the principle of maximum entropy to describe the spatial distributions of the cone cells in the vertebrate eyes and have established a parameter, called collective sensing, which is conserved throughout different species as diverse as: rodent, dog, monkey, human, fish, and bird, regardless of the details of the underlying mechanisms.

Lemaitre's law, which relates the fraction of hexagons to the width of the polygon distribution in numerous two-dimensional cellular mosaics in nature and is usually obtained by assuming an ad hoc constraint, here is derived as a special case of our formalism.

Since we have considered a completely general constraint in the entropy maximization procedure, our formalism could be used to describe other patterns or processes in nature. In the case of failure, it implies that either additional information, which stems from the knowledge of the underlying mechanisms, need to be considered, or the assumed information is incorrect. This indicates that, although in many cases as in this paper, we can describe and predict the phenomena without knowing the details of the underlying dynamics, however, the principle of maximum entropy could still lead us to a better understanding of the involved mechanisms by validating the assumed information. 


\section{Acknowledgments}

I am grateful to Carlo Beenakker and Iosif Pinelis for illuminating and inspiring discussions. I thank the Centre for Information Services and High Performance Computing at TU Dresden for providing an excellent infrastructure.

\section{Conflicts of interest}

The author declares no conflict of interest.

\section{References}

[1] E. T. Jaynes, Information Theory and Statistical Mechanics, Phys. Rev. 106, 620-630 (1957).

[2] E. T. Jaynes, Information Theory and Statistical Mechanics. II, Phys. Rev. 108, 171-190 (1957).

[3] A. Caticha, Lectures on Probability, Entropy, and Statistical Physics, arXiv:0808.0012 (2008).

[4] J. R. Banavar, A. Maritan, and I. Volkov, Applications of the principle of maximum entropy: from physics to ecology, J. Phys.: Condens. Matter 22, 063101 (2010).

[5] T. Mora, A. M. Walczak, W. Bialek, and C. G. Callan, Maximum entropy models for antibody diversity, PNAS 107, 5405-5410 (2010).

[6] J. Harte, T. Zillio, E. Conlisk, and A. B. Smith, Maximum Entropy and the State-Variable Approach to Macroecology, Ecology 89, 2700-2711 (2008).

[7] A. L. Berger, S. A. Della Pietra, and V. J. Della Pietra, A Maximum Entropy Approach to Natural Language Processing, Comput. Linguist. 22, 39-71 (1996).

[8] A. Caticha and R. Preuss, Maximum entropy and Bayesian data analysis: Entropic prior distributions, Phys. Rev. E 70, 046127 (2004).

[9] D. W. Thompson, On Growth and Form, 2nd ed. (Cambridge University Press, Cambridge, 1963).

[10] R. Farhadifar, J. C. Röper, B. Aigouy, S. Eaton, and F. Jülicher, The Influence of Cell Mechanics, Cell-Cell Interactions, and Proliferation on Epithelial Packing, Curr. Biol. 17, 2095-2104 (2007).

[11] H. Wässle and H. J. Riemann, The mosaic of nerve cells in the mammalian retina, Proc. R. Soc. Lond. B Biol. Sci. 200, 441-461 (1978). 
[12] L. Peichl, Diversity of mammalian photoreceptor properties: Adaptations to habitat and lifestyle?, Anat. Rec. A Discov. Mol. Cell. Evol. Biol. 287, 1001-1012 (2005).

[13] K. Viets, K. C. Eldred, and R. J. Johnston, Mechanisms of Photoreceptor Patterning in Vertebrates and Invertebrates, Trends Genet. 32, 638-659 (2016).

[14] R. K. Pathria and P. D. Beale, Statistical Mechanics, 3rd ed. (Elsevier, New York, 2011).

[15] A. Barua, A. Beygi, and H. Hatzikirou, Close to Optimal Cell Sensing Ensures the Robustness of Tissue Differentiation Process: The Avian Photoreceptor Mosaic Case, Entropy 23, 867 (2021).

[16] J. Lemaître, A. Gervois, J. P. Troadec, N. Rivier, M. Ammi, L. Oger, and D. Bideau, Arrangement of cells in Voronoi tessellations of monosize packing of discs, Phil. Mag. B 67, 347-362 (1993).

[17] A. Gervois, J. P. Troadec, and J. Lemaître, Universal properties of Voronoi tessellations of hard discs, J. Phys. A: Math. Gen. 25, 6169-6177 (1992).

[18] W. von der Linden (Ed.), V. Dose (Ed.), R. Fischer (Ed.), and R. Preuss (Ed.), Maximum Entropy and Bayesian Methods (Springer, Dordrecht, 1999).

[19] D. O. Morley, A. L. Thorneywork, R. P. A. Dullens, and M. Wilson, Generalized network theory of physical two-dimensional systems, Phys. Rev. E 101, 042309 (2020).

[20] G. Le Caër and R. Delannay, Correlations in topological models of 2D random cellular structures, J. Phys. A: Math. Gen. 26, 3931-3954 (1993).

[21] P. Cerisier, S. Rahal, and N. Rivier, Topological correlations in BénardMarangoni convective structures, Phys. Rev. E 54, 5086-5094 (1996).

[22] M. P. Miklius and S. Hilgenfeldt, Analytical Results for Size-Topology Correlations in 2D Disk and Cellular Packings, Phys. Rev. Lett. 108, 015502 (2012).

[23] M. Castro, R. Cuerno, M. M. García-Hernández, and L. Vázquez, PatternWavelength Coarsening from Topological Dynamics in Silicon Nanofoams, Phys. Rev. Lett. 112, 094103 (2014).

[24] F. T. Lewis, The correlation between cell division and the shapes and sizes of prismatic cells in the epidermis of cucumis, Anat. Rec. 38, 341-376 (1928).

[25] C. Knessl, Integral representations and asymptotic expansions for Shannon and Renyi entropies, Appl. Math. Lett. 11, 69-74 (1998). 
[26] A. Roorda and D. R. Williams, The arrangement of the three cone classes in the living human eye, Nature 397, 520-522 (1999).

[27] M. P. Wand, Data-Based Choice of Histogram Bin Width, Am. Stat. 51, 59-64 (1997).

[28] D. W. Scott, On optimal and data-based histograms, Biometrika 66, 605610 (1979).

[29] C. Duyckaerts and G. Godefroy, Voronoi tessellation to study the numerical density and the spatial distribution of neurones, J. Chem. Neuroanat. 20, 83-92 (2000).

[30] F. Zhang, K. Kurokawa, A. Lassoued, J. A. Crowell, and D. T. Miller, Cone photoreceptor classification in the living human eye from photostimulationinduced phase dynamics, PNAS 116, 7951-7956 (2019).

[31] F. A. Rocha, P. K. Ahnelt, L. Peichl, C. A. Saito, L. C. Silveira, and S. M. De Lima, The topography of cone photoreceptors in the retina of a diurnal rodent, the agouti (Dasyprocta aguti), Vis. Neurosci. 26, 167-175 (2009).

[32] D. Klein, A. Mendes-Madeira, P. Schlegel, F. Rolling, B. Lorenz, S. Haverkamp, and K. Stieger, Immuno-Histochemical Analysis of Rod and Cone Reaction to RPE65 Deficiency in the Inferior and Superior Canine Retina, PLoS ONE 9, e86304 (2014).

[33] A. Roorda, A. B. Metha, P. Lennie, and D. R. Williams, Packing arrangement of the three cone classes in primate retina, Vision Res. 41, 1291-1306 (2001).

[34] W. T. Allison, L. K. Barthel, K. M. Skebo, M. Takechi, S. Kawamura, and P. A. Raymond, Ontogeny of cone photoreceptor mosaics in zebrafish, J. Comp. Neurol. 518, 4182-4195 (2010).

[35] Y. A. Kram, S. Mantey, and J. C. Corbo, Avian Cone Photoreceptors Tile the Retina as Five Independent, Self-Organizing Mosaics, PLoS ONE 5, e8992 (2010). 